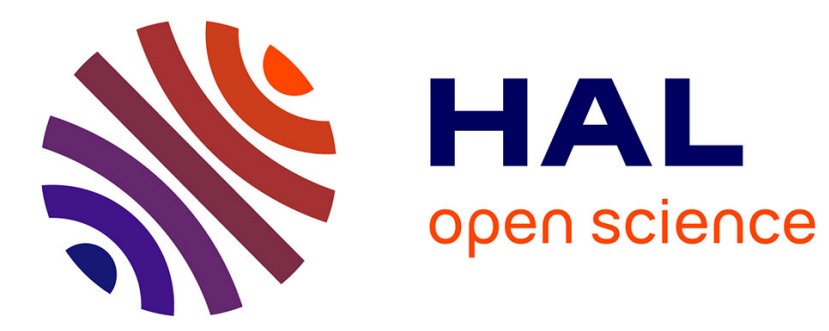

\title{
Purely spatial coincidences of twin photons in parametric spontaneous down-conversion
}

\author{
J.-L. Blanchet, F. Devaux, L. Furfaro, E. Lantz
}

\section{To cite this version:}

J.-L. Blanchet, F. Devaux, L. Furfaro, E. Lantz. Purely spatial coincidences of twin photons in parametric spontaneous down-conversion. Physical Review A : Atomic, molecular, and optical physics [1990-2015], 2010, 81 (4), pp.043825. 10.1103/PhysRevA.81.043825 . hal-00474430

\section{HAL Id: hal-00474430 \\ https://hal.science/hal-00474430}

Submitted on 13 May 2021

HAL is a multi-disciplinary open access archive for the deposit and dissemination of scientific research documents, whether they are published or not. The documents may come from teaching and research institutions in France or abroad, or from public or private research centers.
L'archive ouverte pluridisciplinaire HAL, est destinée au dépôt et à la diffusion de documents scientifiques de niveau recherche, publiés ou non, émanant des établissements d'enseignement et de recherche français ou étrangers, des laboratoires publics ou privés. 


\title{
Purely spatial coincidences of twin photons in parametric spontaneous down-conversion
}

\author{
Jean-Luc Blanchet, Fabrice Devaux, Luca Furfaro, and Eric Lantz \\ Département d'Optique P.M. Duffieux, Institut FEMTO-ST, UMR CNRS 6174, Université de Franche-Comté, \\ 16 route de Gray, F-25030 Besançon Cedex, France \\ (Received 25 January 2010; published 19 April 2010)
}

\begin{abstract}
We have measured sub-shot-noise quantum correlations of spatial fluctuations in the far-field image of the parametric fluorescence created in a type I $\beta$-barium-borate nonlinear crystal, either between opposite angular sectors (nondegenerate configuration) or between opposite pixels (degenerate configuration). Imaging is performed at a very low light level ( 0.2 photons per pixel) with an electron-multiplying CCD camera, resulting in purely spatial coincidences between single photons when detecting on pixels. Experimental results overcome the standard quantum-limit-shot-noise level without subtraction of the variance of the detection noise. We compare these experimental results with numerical results given by the quantum Green's function method, which is proved to have strong advantages over stochastic simulations.
\end{abstract}

DOI: 10.1103/PhysRevA.81.043825 PACS number(s): 42.65.Lm, 42.50.Ar, 42.50.Lc, 42.50.Dv

\section{INTRODUCTION}

Spontaneous down-conversion (SPDC) occurs in a nonlinear crystal when a pump photon splits into a pair of signal and idler photons. Even if the number of pairs fluctuates, this relation is exact in the sense that, in the absence of input light at the signal and idler frequency, the difference between the signal and idler output photon numbers is zero in an ideal experiment. Heidmann et al. [1] showed that the spectrum of temporal fluctuations of the intensity difference between spatially monomode twin beams is below the standard shot-noise level. Actually, the beams are entangled: The phases of the beams are also correlated at the quantum level, as shown by homodyne detection. If the two detectors do not intercept the whole beams, the correlation is reduced because for some pairs one photon is detected while the other is not intercepted. Because "intercepted" can be replaced with "detected" in the previous sentence, insufficient detector size is exactly equivalent to a reduction in the quantum efficiency.

The situation is different for a strongly spatially multimode beam issued from a traveling wave amplifier: Brambilla et al. showed theoretically [2] that, for unity quantum efficiency, the variance of the signal-idler photon number difference goes to zero if the pixel size is much greater than the coherence area. These results were numerically confirmed either by stochastic simulations corresponding to the Wigner formalism [3] or by using the quantum Green's function method [4]. Indeed, Boyer et al. studied temporal fluctuations of spatially broad-band twin beams obtained with four-wave mixing in a hot atomic vapor and showed that part of the beams larger than the coherence area exhibit sub-shot-noise intensity differences [5], as well as entanglement [6], if detected with local oscillators shaped as the beams. These experiments demonstrated temporal entanglement of "subbeams" but did not consider fluctuation of spatial variables, like position or angle. Entanglement of such variables for beams [7] was demonstrated by combining TEM00 beams with a vacuum-squeezed TEM01 beam and homodyne detection of temporal fluctuations [8]. On the other hand, Boyer et al.

\footnotetext{
*eric.lantz@univ-fcomte.fr
}

[9] demonstrated spatial entanglement of photon pairs in an image by varying the position of detectors in both the near and the far field and recording temporal coincidences. Other spatial properties of twin photons have been extensively studied in the group of Boston [10] by recording temporal coincidences.

Though dealing with spatial aspects of multimode beams, all the experiments in the preceding references were devoted to the characterization of temporal fluctuations or temporal coincidences. However, patterns in an image are pure spatial information, without any time aspect, which are ultimately degraded by spatial fluctuations of quantum origin for very weak images [11]. Jedrkiewicz et al. [12] performed the first experimental demonstration of sub-shot-noise behavior of spatial fluctuations of the signal-idler difference. They imaged SPDC issued from a type II $\beta$-barium-borate (BBO) crystal onto a back-illuminated CCD camera and showed that the value of the variance of the difference between signal-idler intensities on opposite pixels is below the shot-noise level. However, this result was obtained by subtracting the variance of the readout noise, that is, about 100 squared photoelectrons, from a measured variance of 110 squared photoelectrons. With a conventional $\mathrm{CCD}$, diminishing the relative weight of the detection noise requires the acquisition of more intense images and convincing results have been recently obtained without subtraction of the background noise [13], for intensities around 600 photons per pixel and a pump pulse duration in the ns range, in order to avoid excess noise due to the thermal character of SPDC [14].

We chose the opposite direction for obtaining sub-shotnoise correlations, without subtraction of the variance of the detector noise, by detecting single photons in low-light-level images with an electron-multiplying CCD camera (EMCCD). In such cameras, the readout noise is rendered negligible by adding a register where the photoelectrons are multiplied before reading. Hence, even a unique photon gives a signal that emerges from the readout floor. However, the gain is stochastic, as in an avalanche photodiode, and it is not possible to assign a precise number of photons to each value of the output signal. It can be demonstrated [15] that dividing the output signal by the mean gain results in adding a Poisson detection noise having the same amplitude as the standard photon noise. This 
excess noise prevents any attempt to detect sub-shot-noise correlations, at least without subtraction of the variance of the detection noise. On the other hand, detection of single photons by thresholding adds in principle no noise for high gain, even stochastic, and very-low-light level images. In practice, false detections occur whose number can be minimized [16] by choosing an appropriate fluence (about 0.15 photon/pixel with our camera) and by adjusting the threshold. Under these conditions, the variance of the detection noise is much smaller than the mean fluence and detection of sub-shot-noise correlations becomes possible. We first studied [17] type I broad-band nondegenerate SPDC and showed correlations between angular sectors, then added an interferential filter to obtain SPDC around degeneracy, in order to obtain correlations between opposite pixels [18]. We proved in this latter case spatial coincidences between individual photons. The aim of this article is to present in more detail these experimental results, especially those in the degeneracy configuration, and to add a discussion of the numerical methods that allow a comparison. In particular, we will show that usual stochastic simulations, based on the Wigner formalism, are not feasible in practice for intensities on the order of tenths of photons per pixel, while the Green's function method can be rendered less computationally expensive than expected at a first sight. The paper is organized as follows. Section II deals with experimental results at degeneracy, while Sec. III treats the broad-band configuration. Section IV is devoted to numerical simulations and Sec. V concludes.

\section{MEASUREMENT OF SUB-SHOT-NOISE CORRELATIONS BETWEEN PIXELS}

The experimental setup is sketched in Fig. 1. The pump pulse provided by the fourth harmonic (0.93-ps duration at $263.8 \mathrm{~nm}$ ) of a $Q$-switched mode-locked $\mathrm{Nd}$ :glass laser (Twinkle laser by Light Conversion Inc.) at a repetition rate of $33 \mathrm{~Hz}$, illuminated a type I $7 \times 7 \times 4-\mathrm{mm}^{3} \mathrm{BBO}$ nonlinear crystal. The far-field image of the parametric fluorescence was formed in the focal plane of a lens by a back-illuminated EMCCD camera from Andor Technology (Model iXon+ DU897-ECS-BV) with a quantum efficiency greater than $90 \%$ in the visible range. The detector area is formed by $512 \times$ 512 pixels, with a pixel size of $16 \times 16 \mu \mathrm{m}^{2}$. We used a readout rate of $10 \mathrm{MHz}$ at 14 bits and the camera was cooled to $-85^{\circ} \mathrm{C}$. The exposure time was $33 \mathrm{~ms}$ and the EM gain was set to 1000 . In these conditions, the readout noise has a standard deviation of 46 electrons and the level of clock-induced noise,

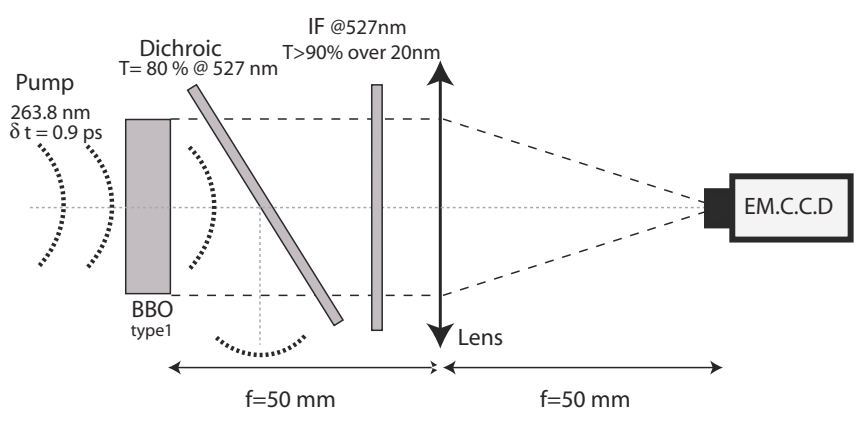

FIG. 1. Experimental setup. that is, generation of spurious electrons during the transfer, is on the order of $4 \times 10^{-3} e^{-}$/pixel. A threshold set to 2.8 readout noise standard deviations allows the number of false detections to be minimized [16]. To eliminate the residual UV, two dichroic filters with a nominal transmission of $95 \%$ at $527 \mathrm{~nm}$ were placed after the BBO crystal. To obtain degenerate parametric fluorescence, an interferential filter (IF) was placed after the dichroics, with a quantum efficiency greater than $90 \%$ over a bandwidth of $20 \mathrm{~nm}$, while broadband fluorescence was obtained simply by removing this filter. The trajectory of the light after the dichroics and the filter was enclosed in a tube in order to avoid parasitic reflections. The energy of the 263.75-nm pump pulse was measured at $106 \pm 38 \mathrm{~nJ}$. The total quantum efficiency is the product of the quantum efficiency of the EMCCD by the transmission of the optical elements after the crystal:

$$
\eta_{\mathrm{tot}}=\eta_{\mathrm{CCD}} \times \eta_{\mathrm{opt}} \times \eta_{\mathrm{IF}}=0.9 \times 0.68 \times 0.9=0.55 .
$$

$\eta_{\mathrm{CCD}}$ is given by the manufacturer and $\eta_{\mathrm{opt}}$ was measured. In particular, a transmission by the two dichroic filters of $80 \%$ has been measured.

Measurements at degeneracy were performed for a crystal orientation corresponding to collinear phase matching. Figure 2 shows a sum of 50 single-shot images of parametric fluorescence recorded by the EMCCD. Unlike in a single image, the fluorescence disk is clearly visible. The mean level in the disk for one image, about 0.20 photon per pixel, has been chosen in order to minimize the number of false detections [16].

We have measured the difference between the number of photons in opposite pixels, which should go to zero for a perfect detector, perfect degeneracy, and negligible diffraction, that is, for a coherence area much smaller than the pixel size [4]. This last condition is fulfilled here because of the wide illumination of the crystal: The measured pump width on the crystal [full width at half maximum (FWHM)] is $3.2 \mathrm{~mm}$. For

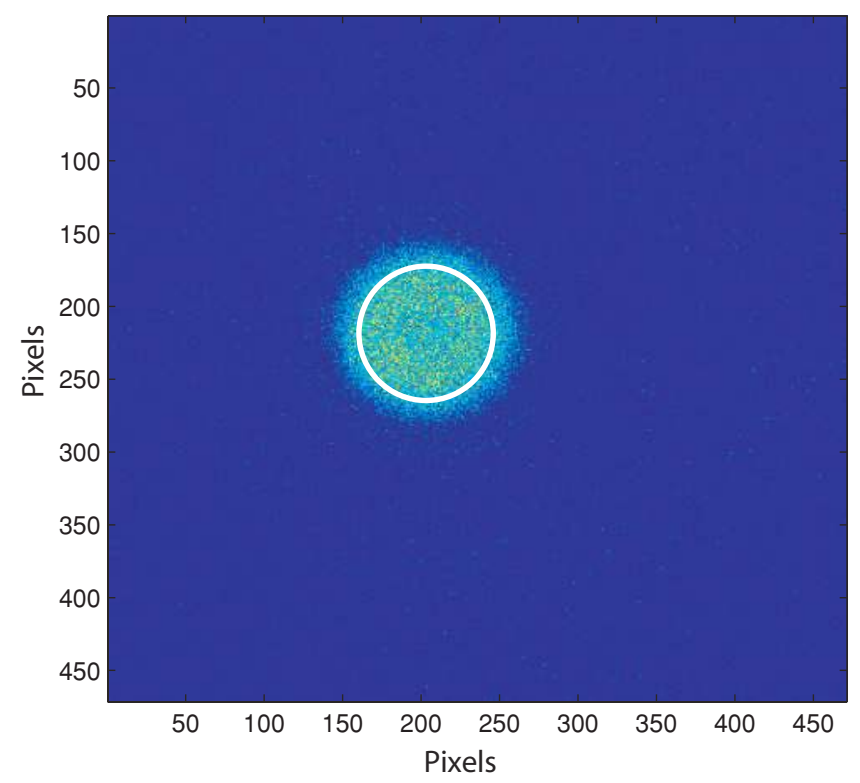

FIG. 2. (Color online) Sum of 50 experimental images. The white circle delimits the area where the statistic is performed. 
pure SPDC with negligible further amplification and a pump beam area smaller than the crystal section $\left(7 \times 7 \mathrm{~mm}^{2}\right.$ here $)$, the down-converted beam has the same intensity profile as the pump beam. The width of the coherence area in the far field, $0.07 \mathrm{mrad}$ (FWHM), is proportional to the inverse of the width of this beam [19] and is much smaller than the 0.32-mrad lateral size of the CCD pixel. Moreover, the mean number of photons for one spatiotemporal mode is less than $10^{-2}$, resulting in theoretical Bose-Einstein photon distribution [20] that is undistinguishable from a Poisson distribution. Indeed, the number of amplified temporal modes is approximately 40 [19] and the number of amplified spatial modes on a pixel is on the order of $(0.32 / 0.07)^{2} \simeq 20$. To take into account the nonuniform level of the electronic background over the detector area, this background is measured in darkness and subtracted from the SPDC images in the form of a mean square plane. Then, a thresholding procedure is applied on each image in order to decide whether there is one or zero photoelectrons on each pixel [16]. Note that it is not possible to distinguish the (rare) cases where two photoelectrons are present on one pixel. The variance of the difference between opposite pixels is then computed inside a circle containing $N$ pixels where the level of SPDC is approximately constant. The center of this circle is determined by minimizing the variance of the difference

$$
\sigma_{\text {diff }}^{2}=\frac{1}{N / 2} \sum_{i=1}^{N / 2}\left(n_{i}-n_{N-i}\right)^{2},
$$

with a numbering of the pixels ensuring that indices $i$ and $N-i$ correspond to opposite pixels. The center determination must be performed with precision [14], at best with a resolution of half a pixel in order to keep the actual pixels with, if necessary, an unused crux (see Fig. 3). After this centering has been performed with physical pixels, statistics can be calculated using square blocks of pixels called binned pixels.

It should be noted that subpixel centering algorithms exist [21,22] and have been employed with good results in [13], but we have not used them in the presented results in order to conserve a measurement of either zero or one photon per physical pixel. We have nevertheless verified that improvements of results provided by these methods do exist for our experimental results but are weak because of the predominence of quantum noise in our low-flux regime.
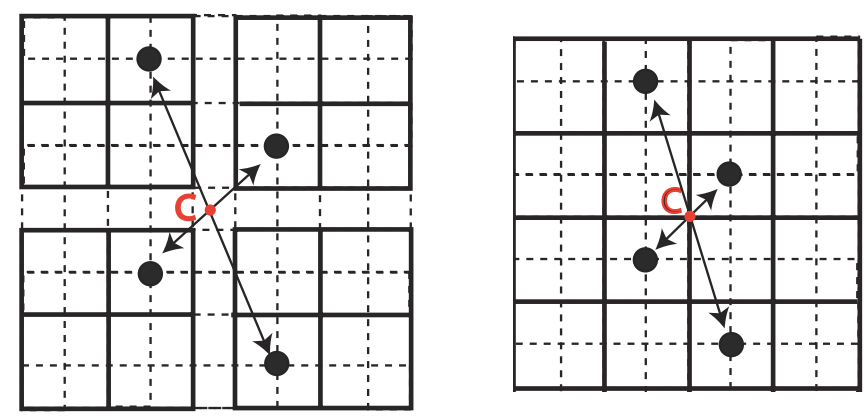

FIG. 3. (Color online) Centering for a $2 \times 2$ binning. (Left) The best center corresponds to the center of a physical pixel. (Right) The best center corresponds to the corner between four physical pixels.
For perfect detection, sub-shot-noise correlations exist if $\sigma_{\text {diff }}^{2}$ is smaller than twice the mean $n_{\text {moy }}=\frac{1}{N} \sum_{i=1}^{N}\left(n_{i}\right)$. However, the measured variance of the photon number appears to be smaller than the mean photon number, while the equality is expected for a Poisson distribution. This phenomenon can be easily explained by taking into account the cases where two or more photoelectrons are accumulated in the same pixel. If $\mu$ is the true mean number of photoelectrons accumulated in one pixel, a thresholding procedure would give, in the absence of false detections, a measured mean $m$ given by

$$
m=1-p(0)=1-\exp (-\mu),
$$

where $p(0)$ is the probability of detecting no photoelectron. The first equality expresses the fact that the thresholding procedure is unable to distinguish between one and more photoelectrons on one pixel, while the second equality reflects the Poisson distribution of photoelectrons. With the same hypotheses, the measured variance $\sigma^{2}$ is given by

$$
\sigma^{2}=m^{2} p(0)+(1-m)^{2}[1-p(0)]=m(1-m) .
$$

Hence, the measured variance is smaller than the measured mean, because of the binary detection. On the other hand, the variance of the difference is affected in the same way as the variance by this effect. To cancel this artifact, the criterion for the detection of sub-shot-noise correlations becomes

$$
\frac{\sigma_{\text {diff }}^{2}}{m(1-m)} \leqslant 2 \text {. }
$$

Hence, the measured ratio $\sigma_{\text {diff }}^{2} / n_{\text {moy }}$ must be multiplied by a correction coefficient $c=1 /\left(1-n_{\text {moy }}\right)$ in order to be compared to the shot-noise limit (SNL). If binned pixels are used, $c$ must be estimated before binning, since thresholding is performed on the physical pixels.

Figure 4 presents the measured ratios on 50 images, without binning and with 4848 physical pixels in the statistics area or for 1212 blocks of $2 \times 2$ pixels. In the absence of binning, the corrected ratios $r$ are almost exactly equal to the ratios of the variances $r^{\prime}=\sigma_{\text {diff }}^{2} / \sigma^{2}$, meaning that the classical noise is negligible, while the corrected ratios are slightly different from the variance ratios for $2 \times 2$ binning, because of the smaller number of samples. In both cases, these ratios are clearly in the quantum regime. At $95 \%$ of confidence the results on individual images are

$$
\begin{gathered}
r=c \frac{\sigma_{\text {diff }}^{2}}{n_{\text {moy }}}=1.94 \pm 0.08, \\
r^{\prime}=\frac{\sigma_{\text {diff }}^{2}}{\sigma_{s}^{2}}=1.94 \pm 0.08
\end{gathered}
$$

for physical pixels and

$$
\begin{gathered}
r=c \times \frac{\sigma_{\text {diff }}^{2}}{n_{\text {moy }}}=1.82 \pm 0.18, \\
r^{\prime}=\frac{\sigma_{\text {diff }}^{2}}{\sigma_{s}^{2}}=1.83 \pm 0.16
\end{gathered}
$$

for blocks of $2 \times 2$ pixels. In this latter case, the dispersion is doubled, because the number of pixels has been divided by 4. While the limits of the confidence interval for individual 

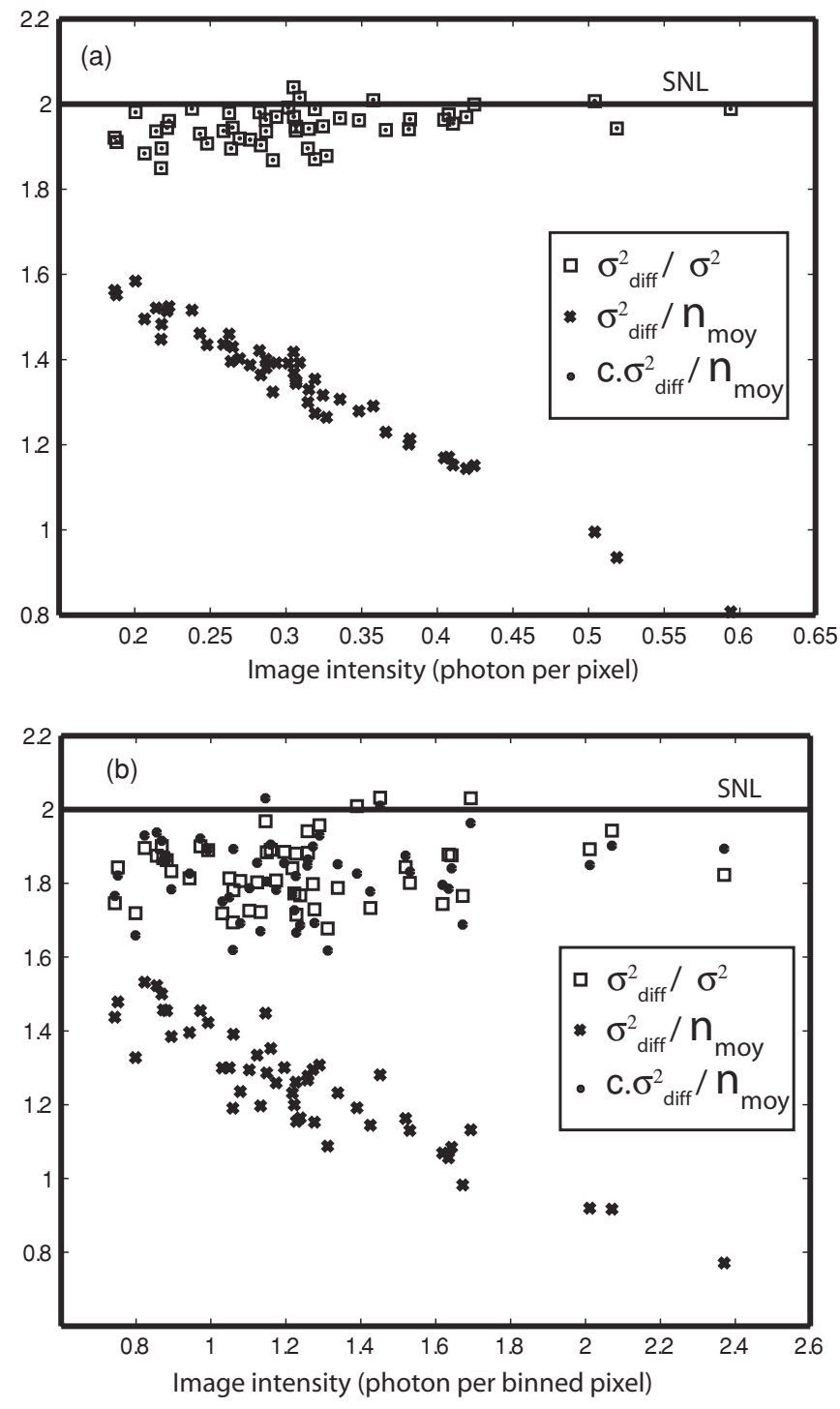

FIG. 4. Experimental results. Each point corresponds to a singleshot measurement. (a) No binning; (b) $2 \times 2$ binning.

images attain the SNL, the averages of the estimators on the 50 images are well below the SNL,

$$
\begin{aligned}
& \langle r\rangle=1.94 \pm \frac{0.08}{\sqrt{5} 0}=1.94 \pm 0.01, \\
& \left\langle r^{\prime}\right\rangle=1.94 \pm \frac{0.08}{\sqrt{5} 0}=1.94 \pm 0.01
\end{aligned}
$$

for physical pixels and

$$
\begin{aligned}
& \langle r\rangle=1.82 \pm \frac{0.18}{\sqrt{5} 0}=1.82 \pm 0.02, \\
& \left\langle r^{\prime}\right\rangle=1.83 \pm \frac{0.18}{\sqrt{5} 0}=1.83 \pm 0.02
\end{aligned}
$$

for blocks of $2 \times 2$ pixels.

To conclude this section, we have demonstrated pure spatial quantum correlations between opposite pixels of different sizes. For the smallest size, corresponding to the physical pixels, this correlation corresponds to spatial coincidences between individual photons, because the number of photons per pixel is either one or zero. However, though diffraction is negligible even for this pixel size, correlations are reduced because of imperfect centering, nonperfect degeneracy, and detector errors, as is shown in Sec. IV. The best results have been obtained by grouping the pixels in $2 \times 2$ blocks.

\section{MEASUREMENT OF SUB-SHOT-NOISE CORRELATIONS BETWEEN ANGULAR SECTORS}

We now describe results obtained without chromatic filtering for a crystal orientation corresponding to noncollinear phase matching. Figure 5 shows a sum of 58 single-shot images in this configuration. For nondegenerate wavelengths, the idler and signal fluorescence form rings of different diameter and the rings corresponding to different wavelengths add incoherently in the image. However, because of momentum conservation, each pair of twin photons emitted in the SPDC process, although not equidistant from the center of the pattern, lies along a diameter line, as shown in Fig. 6.

The SPDC image is divided in $S=90$ angular sectors and a number of photons $n_{i}$ is determined in the intersection of each of these sectors with a ring encompassing the greatest part of the multimode SPDC. The center of this ring is determined in order to obtain the most regular distribution of light between sectors on the sum image. Note that only the pump beam experiences walk-off: The center of the SPDC ring does not correspond to the center of the pump beam, with no practical consequences since this pump beam is not detected. The size of a sector, 240 pixels, results from a compromise between effects of diffraction and not perfect centering, which are more sensitive for small sectors, and of the other classical noises (e.g., deterministic residual aberrations, see later in this article) that predominate if the number of photons in a sector is too large. The symmetrical sector-pair correlation is evaluated by estimating the ratios $r$ and $r^{\prime}$ defined in the

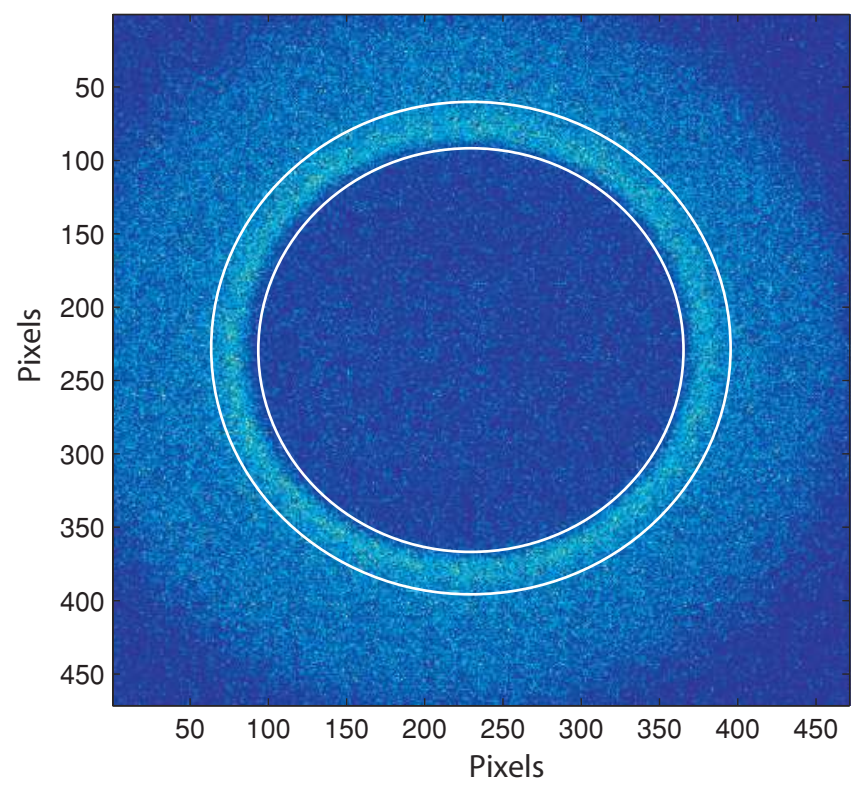

FIG. 5. (Color online) Sum of 58 experimental images in passband configuration. The white circles delimit the area where the statistic is performed. 

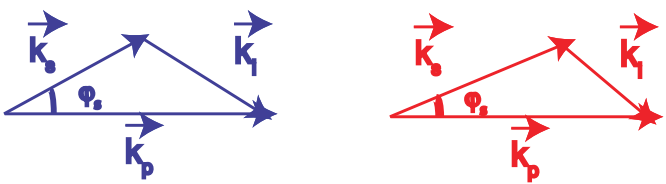

(a) Phase matching condition
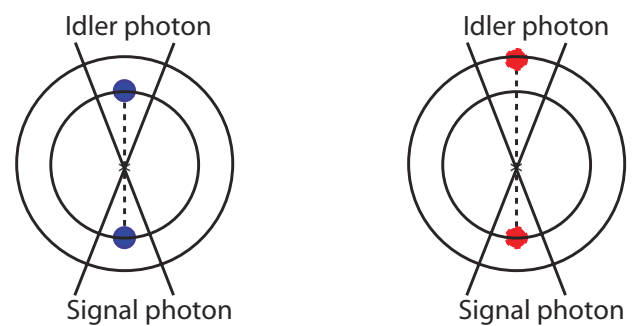

(b) Opposite angular sectors

Degeneracy

Outside degeneracy

FIG. 6. (Color online) (a) Noncollinear phase matching; $\vec{k}_{p, s, i}$, wave vectors of, respectively, pump, signal, and idler. (b) Correlation between angular sectors. For noncollinear phase matching, idler and signal form rings of different diameters.

preceding section. Figure 7 shows the experimental results for 58 single-shot images with a mean comprised between 0.1 and 0.25 photon/pixel: Each point corresponds to a single-shot measurement with a statistics performed over the 90 sectors. Results can be summarized for the whole set of images as

$r=c \frac{\sigma_{\text {diff }}^{2}}{n_{\text {moy }}}=1.85 \pm 0.78 \quad r^{\prime}=\frac{\sigma_{\text {diff }}^{2}}{\sigma_{s}^{2}}=1.75 \pm 0.50$.

For both values, the uncertainty range is centered on the average $\bar{r}$ or $\overline{r^{\prime}}$ of the coefficients of the 58 images and the range width, that is, \pm 2 standard deviations of these 58 coefficients, gives a confidence of $95 \%$ for Gaussian measurement errors. The dispersion of the measurements of $r$ is mainly due to the measurement of $\sigma_{\text {diff }}^{2}$ on a limited set of 90 pairs of sectors, giving a theoretical standard deviation for Gaussian

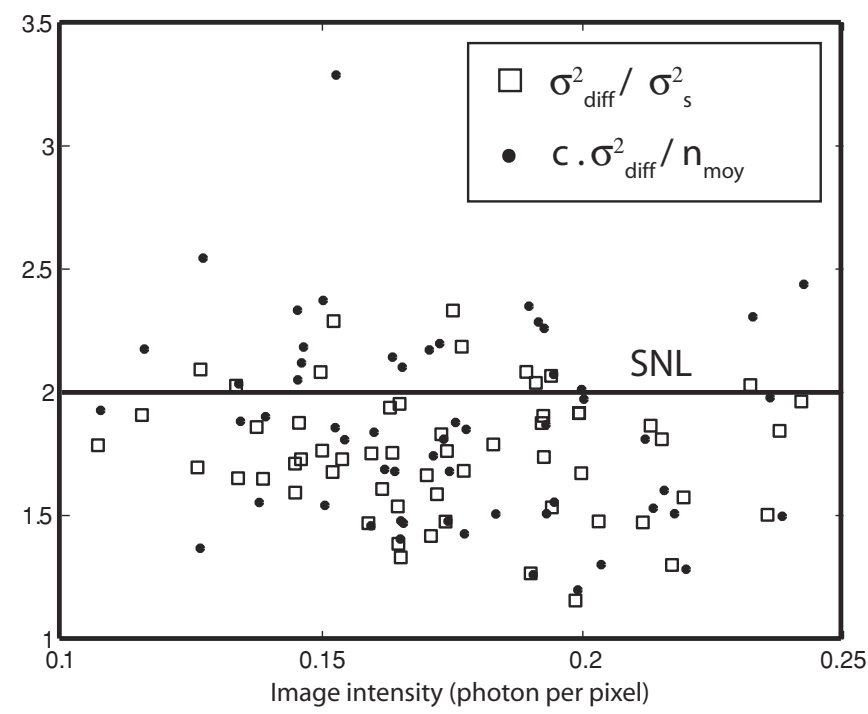

FIG. 7. Experimental results. Each point corresponds to a singleshot measurement. statistics $\sigma_{\mathrm{var}}=(2 / 90)^{1 / 2} \sigma_{\text {diff }}^{2}$, hence a standard deviation on $r$, by neglecting the much smaller uncertainty on $m: \sigma_{r}=$ $(2 / 90)^{1 / 2} r$, that is a theoretical uncertainty range of \pm 0.45 . The other important source of dispersion of $r$ comes from the fluctuations of the mean photon number from an image to another due to the fluctuations of the pump energy. Though some measurement values on individual images are greater than 2, in accordance with the uncertainty range of Eq. (13), the mean coefficients for the 58 images are significantly smaller than 2:

$$
\begin{aligned}
& \langle r\rangle=1.85 \pm \frac{0.78}{\sqrt{58}}=1.85 \pm 0.10 \\
& \left\langle r^{\prime}\right\rangle=1.83 \pm \frac{0.50}{\sqrt{5} 8}=1.83 \pm 0.07 .
\end{aligned}
$$

We show in Fig. 8(b) the evolution of the variance of the photon number difference between two nonopposite sectors,
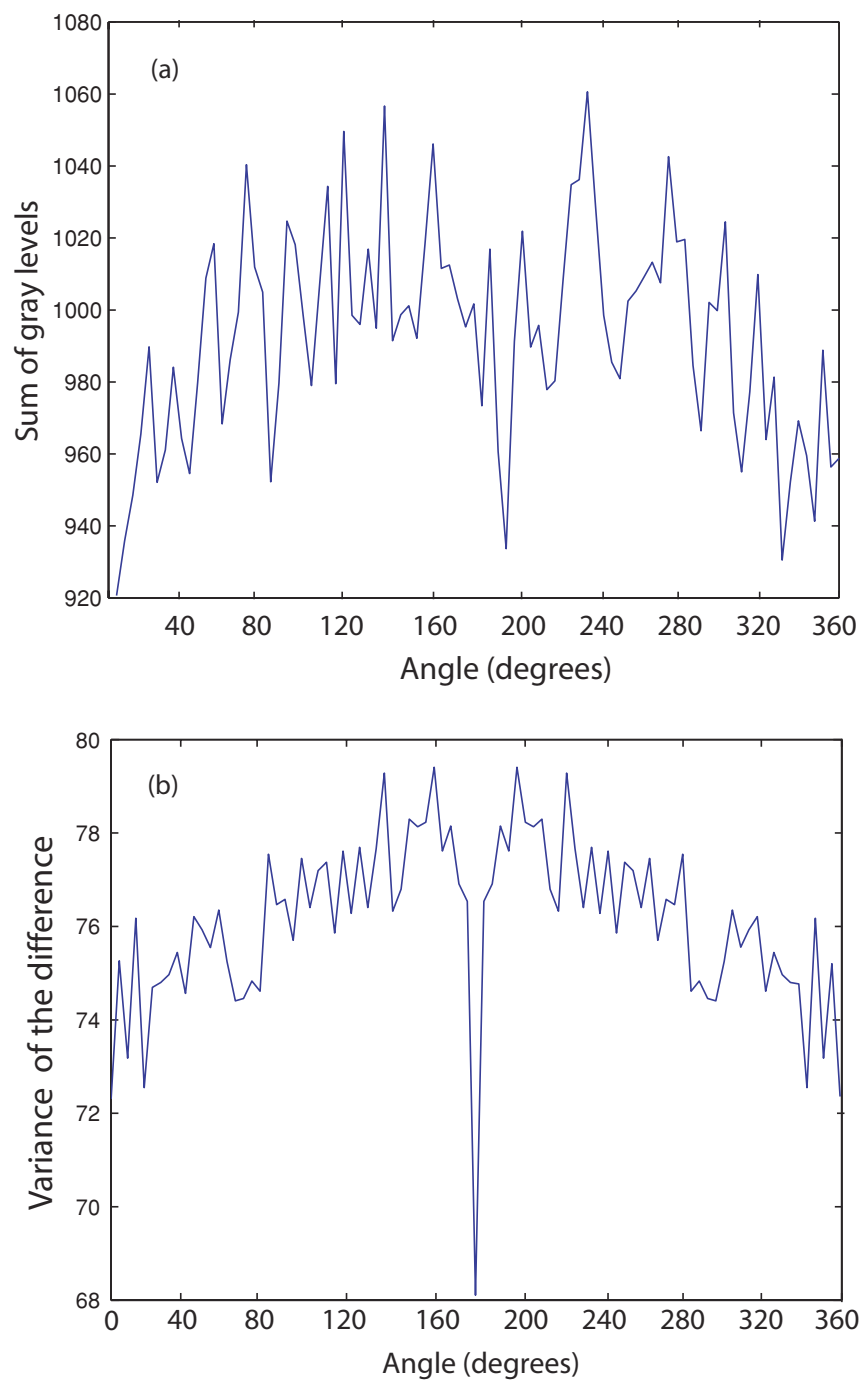

FIG. 8. (Color online) (a) Intensity (in sum of gray levels/sector) of the sum image versus the angular position of the sector. (b) Variance of the difference between sectors versus their angular separation. 
versus their angular separation $\alpha$ :

$$
\sigma_{\mathrm{diff}, \alpha}^{2}=\frac{1}{S / 2-1} \sum_{i=1}^{S / 2}\left(n_{i}-n_{i+\frac{\alpha S}{2 \pi}}\right)^{2} .
$$

This variance increases when increasing $\alpha$, because of some residual deterministic aberrations which are evidenced on Fig. 8(a), and falls abruptly for opposite sectors, because of quantum correlations. Nevertheless, these deterministic aberrations deteriorate the quantum correlations. Another deterioration comes from noncollinear phase matching in the cases where the signal ring is included in the detection area while the idler ring lies outside this area. If the detection was perfect, such a situation could be avoided by extending the outer diameter of the detection ring. However, in practice, experimental results are worse because of the contribution of the detector noise in the low-intensity part of this area. The other effects which deteriorate the theoretically perfect quantum correlations are detailed in the next section.

\section{NUMERICAL SIMULATIONS}

\section{A. Stochastic simulations}

In the linear approximation, one can show that the Wigner distribution of the output field can be simulated by integrating the classical propagation equations starting from a stochastic field which has the phase-space distribution determined by the input field Wigner function. By averaging a great number of such simulations, one determines the expectation values of symmetrized operators. However, quantities measured in an experiment do not correspond to symmetrized operators but rather to normal ordering, so that correction terms must be added to the averages. First, the expectation of the photon number in pixel $\vec{r}$ is given by

$$
\left\langle\hat{a}_{\vec{r}}^{+} \hat{a}_{\vec{r}}\right\rangle=\left\langle\alpha_{\vec{r}}^{*} \alpha_{\vec{r}}\right\rangle_{\text {stoch }}-\frac{1}{2} .
$$

Similarly, the normally ordered photon number variance is obtained by subtracting $\frac{1}{4}$ to the stochastic variance.

To perform the Monte-Carlo numerical simulations, we proceed with the following steps [4]:

(i) For each temporal mode, we generate the stochastic input field with the appropriate phase-probability distribution corresponding to the vacuum field in the Wigner representation, that is, for each pixel Gaussian white noise with zero mean and a random phase, such that $\left\langle\alpha_{\vec{r}}^{*} \alpha_{\vec{r}}\right\rangle_{\text {stoch }}=\frac{1}{2}$.

(ii) The propagation of the stochastic field is then evaluated by integrating classical propagation equations, which are solved with a split-step algorithm.

(iii) The fluences for each temporal mode are added at the output to obtain results corresponding to a single trajectory.

(iv) The expectations of the symmetrically ordered operators are estimated by averaging the results over a great number of trajectories.

(v) All the expectation values in the normal ordering are finally obtained from these stochastic averages by applying the appropriate corrections.

The duration of a temporal mode is roughly equal to the inverse of the bandwidth of the SPDC, resulting for a pump pulse duration of $1 \mathrm{ps}$ in approximately a temporal mode per $\mathrm{nm}$ of bandwidth, that is, approximately 20 temporal modes for a bandwidth limited by the IF, or 40 temporal modes in the broadband SPDC configuration (the other modes give light outside the statistics area). Each temporal mode must be simulated with its proper couple of wavelengths. The final fluence of the order of 0.15 photons/pixel is thus obtained by subtracting $40 \times \frac{1}{2}=20$ photons/pixel from the averaged output. The variance $\sigma_{\text {before }}^{2}$ of this fluence before corrections, obtained as the average of $N$ trajectories, has a mean of the order $40 \times \frac{1}{4}=10$ photons $^{2} /$ pixel and obeys a Gaussian statistics ( $\chi^{2}$ law with $40 N$ degrees of freedom) and with a variance $\sigma_{\text {before }}^{4} / N$. Hence, if the physical variance, that is, the variance after corrections, is of the order of 0.15 , a huge number of trajectories must be averaged to determine $\sigma_{\text {diff }}^{2}$ with a precision at $95 \%$ of confidence of, say, $10 \%$ :

$$
\frac{\sigma_{\text {before }}^{4}}{N}=\left(0.05 \sigma^{2}\right)^{2} \Rightarrow N=\left(\frac{\sigma_{\text {before }}^{2}}{0.05 \sigma^{2}}\right)^{2} \simeq 1.8 \times 10^{6} \text {. }
$$

This number has to be multiplied by the number of temporal modes. To conclude this section, stochastic simulations need a nonacceptable huge number of runs to give a good precision, because of the low light level in the output image, resulting in a too-high difference between the fluences in the corrected and the noncorrected images.

\section{B. Green's function method}

In the undepleted pump approximation, equations of parametric amplification are linear and the output field on the pixel $\vec{r}$ can be described as the sum of contributions from all the pixels $\overrightarrow{r_{1}}$, multiplied by Green's function $G\left(\vec{r}, \overrightarrow{r_{1}}\right)$. To take into account the noncommuting character of the fields in their quantum description, two Green's functions $G\left(\vec{r}, \vec{r}_{1}\right)$ and $H\left(\vec{r}, \vec{r}_{1}\right)$ must be introduced [23]. Their numerical values are computed using a $\delta$ function successively on both quadratures corresponding either to a maximum amplification or a maximum deamplification as an initial condition in the classical propagation equation. The output quadrature fields obtained through the numerical propagation of this $\delta$-like input functions are directly proportional to linear combinations of the Green's functions. It is then easy to deduce the actual value of $G$ and $H$ after the numerical propagation of a $\delta$ function centered at each point of the transverse plane. To summarize the anterior work [4], the Green's functions $H$ and $G$ can be numerically computed as linear combinations of output fields obtained by propagation of $\delta$-function input fields. The propagation of these input fields must be computed for each position in the output crystal plane and for both input quadratures. The knowledge of these Green's functions allows us to compute all the output covariance functions.

To describe the experiment, the number and the size of the pixels in the simulation must correspond to the actual CCD sensor. At first glance, it means that we have to calculate $G$ and $H$ Green's functions for $2 \times 512^{2}$ input $\delta$ functions, giving for 1 input pixel (two $\delta$ functions, one per quadrature) $2 \times 512^{2}$ output values (two Green's functions $G$ and $H$ ). This scheme seems not practicable because of the half-million simulations and the $10^{11}$ output values. However, 
symmetries and negligible terms allow a considerable reduction of the computations. For example, an angular sector includes 240 pixels. To calculate $\sigma_{\text {diff }}^{2}$ characterizing this sector, simulations must be performed for input Diracs on the pixels of the sector plus a border 2 pixels wide, to take into account diffraction, and for input Diracs on an opposite area of the same dimensions, to retrieve signal-idler correlations. Hence, approximately 2000 propagations of a field of $512 \times$ 512 pixels must be performed and it is necessary to keep in memory only the results for $\vec{r}$ inside the sector or its opposite. This number is even considerably reduced in the degenerate case, because a binned pixel and its border include less than 100 physical pixels. The preceding numbers correspond to one temporal mode and must be multiplied by the number of these modes that give a significant contribution. To conclude this paragraph, the Green's function method appears to be the only one that allows the computation of quantum covariances for very low fluxes corresponding to photon counting detection.

\section{Results}

Four types of cause deteriorate the ideal perfect signal-idler correlation when considering opposite areas. First, the idler photon is detected in a coherence area around the exact opposite position of the detection of the signal photon, because of diffraction. This effect will be quantified by simulations involving only one temporal mode corresponding to degeneracy and oversampling in the far field. Second, the center of a physical pixel or the corner between four pixels does not correspond exactly to the symmetry center of the far-field image. Third, twin photons correspond to nondegenerate frequencies, resulting in a noncollinear phase matching scheme and nonopposite locations in the far field (see Fig. 6). The Green's function allows us to quantify this effect by using a sufficient number of temporal modes, with a specific sampling in the image space (near field) for each in order to obtain a uniform sampling in the far field corresponding to the actual CCD sensor. Fourth, the imperfect detection by the camera leads to the loss of some photons and to the detection of spurious photoelectrons that do not correspond to actual photons. We present in the following results these four types of error.

To quantify diffraction effects, we have to take into account the actual dimensions of the illuminated crystal and of the pump beam in the near field, while the sampling step in this near field must correspond to the entire phase-matching bandwidth in the far field. By using $2048 \times 2048$ samples, both requirements are fulfilled, with $4 \times 4$ samples corresponding to one physical pixel of the camera in the far field. Even for the smallest measurement area, that is, one physical pixel, the effect of diffraction appears to be weak, $r<4 \times 10^{-2}$, because the coherence area is sufficiently smaller than the physical pixel. Hence, the other effects will be simulated with $512 \times 512$ samples, that is, a sample length equal to a fourth of the crystal transversal size, in order to keep computation times reasonable and to obtain in the far field an equal size between the sample in the simulations and the physical pixel. Diffraction effects will be nevertheless taken into account by using a pump beam diameter smaller than its actual diameter in order to retrieve the same value of $r$ as with $2048 \times 2048$ samples.

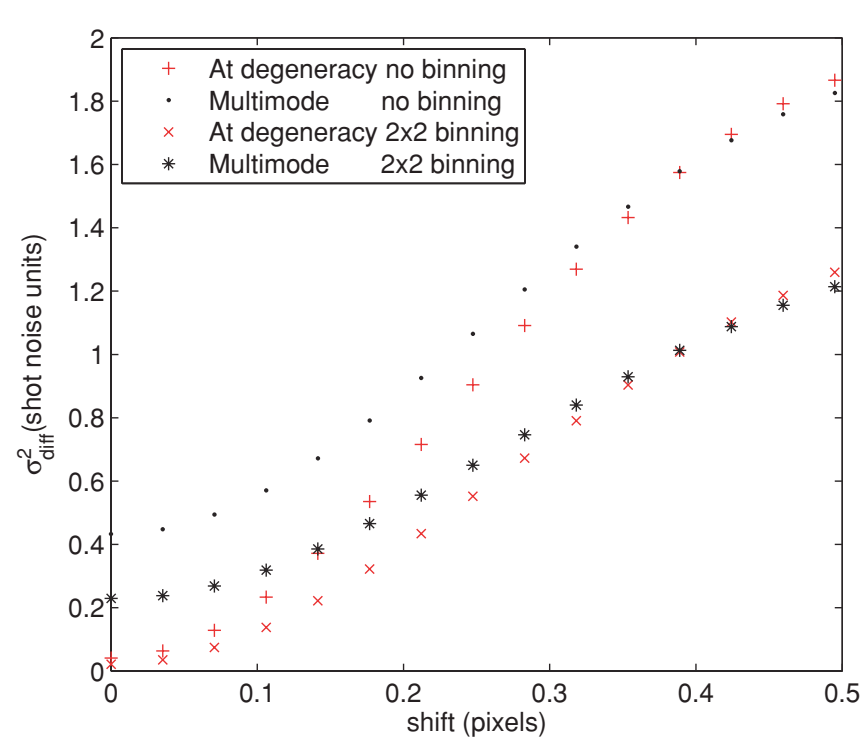

FIG. 9. (Color online) Variance of the signal-idler difference versus the shift between the actual and the used center. The values on the abscissa correspond to a shift along a diameter and to a shift of the same value in the orthogonal direction. The distance between the twin pixels and the center is 20 pixels.

Centering is physically performed with a resolution of half a pixel in order to avoid interpolation (see Sec. II). The maximum difference between the actual center and the center used in calculations of Sec. II is therefore 0.25 pixel, if the actual center is determined without error. Figure 9 shows the evolution of $r$ with this difference in the cases of no binning and $2 \times 2$ binning. For the maximum theoretical shift of 0.25 pixel, $r$ attains half the SNL if no binning is performed and twice as less for $2 \times 2$ binning. $r$ becomes negligible for greater binning, in particular in the case of the angular sectors of Sec. III.

Figure 9 shows also the difference between a strict degeneracy and the experiment described in Sec. II, where the SPDC is rendered narrow-band by a 20 -nm-wide filter. For perfect centering and without binning, $r$ equals 0.4 for this multimode light. Actually, this value depends notably on the position of the opposite pixels that are used for the simulation. If the pair of pixels is close to the center of the far-field fluorescence disk, there is almost no degradation due to the multimode character of the SPDC, as shown in Fig. 10: Involved angles are small and the locations for different wavelengths, though not exactly opposite, are shifted by far less than 1 pixel. However, the shift increases linearly with the distance between both pixels, resulting in a linear increase of $r$. Actually, using a smaller statistics area (maximum distance from the center of 20 pixels instead of 40) induces a diminution of the experimental value of $r: 1.93$ instead of 1.94 found in Eq. (11), but with a greater dispersion between images because of the smaller number of pixels available for the statistics. Note that an error in centering diminishes the effect of nonperfect degeneracy because the asymmetry due to different wavelengths compensates in part the asymmetry due to imperfect centering: See the points for a high centering error in Fig. 9. All the points in this figure have been obtained for twin pixels 20 pixels away from the center, that is, a middle value of this distance. 


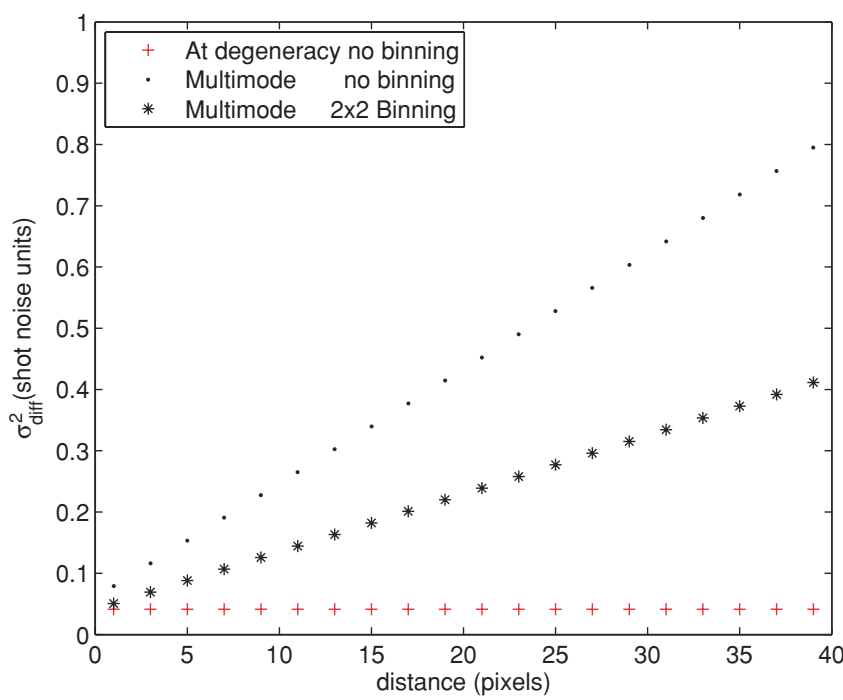

FIG. 10. (Color online) Variance of the signal-idler difference versus the distance between the twin pixels and the center.

In actual measurements, $r$ is computed by using different distances between twin pixels and without knowing the actual error of the center position. To take into account all the parameters, we have repeated the simulation for multimode SPDC with random values of the centering errors between 0 and 0.25 on both axes and random positions of the twin pixels inside the disk. We obtain $r_{\text {perfect }}=0.93$ for perfect detection. We have then computed with our model of EMCCD camera [16] the probability $p_{1}$ of detecting only one photon on one pixel when opposite pixels receive either 0 or twin photons (ideal correlation before detection) for a detected photon mean $m$ [see Eq. (3)] equal to 0.20 , in agreement with the average of experimental results. The variance $\sigma_{\text {diff }}^{2}$ after detection can then be computed as

$$
\sigma_{\text {diff }}^{2}=r_{\text {perfect }} \sigma^{2}+p_{1}\left(1-r_{\text {perfect }} / 2\right),
$$

where $\sigma^{2}$ is computed with Eq. (4). We obtain as a final result for one physical pixel without binning:

$$
r=c \frac{\sigma_{\text {diff }}^{2}}{m}=1.47 .
$$

A part of the remaining difference between experiment ( $r=1.94)$ and simulation $(r=1.47)$ is due to deterministic aberrations, visible on Fig. 8(a). However, this part is weak in the case of correlations between single pixels, because the quantum noise is predominent for a fluence smaller than 1 photon/pixel, and the origin of the discrepancy between simulation and experimental results is not clear. Note however that parametric amplifiers are often described in quantum optics by introducing an "excess noise" factor [24], whose origin comes from distortions in the pump wave front.

In the case of a $2 \times 2$ binning, the experimental and simulated ratios become, respectively, $r=1.82$ and $r=1.29$, while, for angular sectors, they become $r=1.75$ and $r=1.33$. Note that, because of the greater number of photons in an angular sector, the deterministic aberrations induce an increase of $r$ of about 0.1 that is no more negligible.

\section{CONCLUSION}

In conclusion, we have experimentally demonstrated in the photon-counting regime that opposite spatial fluctuations of SPDC radiation are correlated in the quantum regime with a variance of the photon numbers between opposite areas below the shot-noise level. This conclusion holds close to degeneracy for opposite pixels, as well as for broad-band SPDC for opposite angular sectors. In the case of physical pixels, purely spatial coincidences have been demonstrated because the fluence of 0.2 photon/pixel corresponds to either zero or one photon on the pixel. These experimental results are supported by numerical simulations based on the Green's function method, which has been proved to have strong advantages on stochastic simulations for such a low photon flux.
[1] A. Heidmann, R. J. Horowicz, S. Reynaud, E. Giacobino, C. Fabre, and G. Camy, Phys. Rev. Lett. 59, 2555 (1987).

[2] E. Brambilla, A. Gatti, L. Lugiato, and M. Kolobov, Eur. Phys. J. D 15, 127 (2001).

[3] E. Brambilla, A. Gatti, M. Bache, and L. A. Lugiato, Phys. Rev. A 69, 023802 (2004).

[4] E. Lantz, N. Treps, C. Fabre, and E. Brambilla, Eur. Phys. J. D 29, 437 (2004).

[5] V. Boyer, A. M. Marino, and P. D. Lett, Phys. Rev. Lett. 100, 143601 (2008).

[6] V. Boyer, A. M. Marino, R. C. Pooser, and P. D. Lett, Science 321, 544 (2008).

[7] M. T. L. Hsu, W. P. Bowen, N. Treps, and P. K. Lam, Phys. Rev. A 72, 013802 (2005).

[8] K. Wagner, J. Janousek, V. Delaubert, H. Zou, C. Harb, N. Treps, J. F. Morizur, P. K. Lam, and H. A. Bachor, Science 321, 541 (2008).
[9] J. C. Howell, R. S. Bennink, S. J. Bentley, and R. W. Boyd, Phys. Rev. Lett. 92, 210403 (2004).

[10] D. V. Strekalov, A. V. Sergienko, D. N. Klyshko, and Y. H. Shih, Phys. Rev. Lett. 74, 3600 (1995).

[11] A. Mosset, F. Devaux, and E. Lantz, Phys. Rev. Lett. 94, 223603 (2005).

[12] O. Jedrkiewicz, Y.-K. Jiang, E. Brambilla, A. Gatti, M. Bache, L. A. Lugiato, and P. Di Trapani, Phys. Rev. Lett. 93, 243601 (2004).

[13] G. Brida, L. Caspani, A. Gatti, M. Genovese, A. Meda, and I. R. Berchera, Phys. Rev. Lett. 102, 213602 (2009).

[14] E. Brambilla, L. Caspani, O. Jedrkiewicz, L. A. Lugiato, and A. Gatti, Phys. Rev. A 77, 053807 (2008).

[15] J. Hynecek and T. Nishiwaki, IEEE Trans. Electron Devices 50, 239 (2003).

[16] E. Lantz, J.-L. Blanchet, L. Furfaro, and F. Devaux, Mon. Not. R. Astron. Soc. 386, 2262 (2008). 
[17] J.-L. Blanchet, F. Devaux, L. Furfaro, and E. Lantz, Phys. Rev. Lett. 101, 233604 (2008).

[18] J. L. Blanchet, E. Lantz, L. Furfaro, and F. Devaux, CLEO/Europe-EQEC, ICM Munich, Germany, 14-19 June 2009, paper EA.P.2 WED.

[19] F. Devaux and E. Lantz, Eur. Phys. J. D 8, 117 (2000).

[20] A. Mosset, F. Devaux, G. Fanjoux, and E. Lantz, Eur. Phys. J. D 28, 447 (2004)
[21] L. Oriat and E. Lantz, Pattern Recognit. 31, 761 (1998).

[22] L. Caspani, O. Jedrkiewicz, E. Brambilla, and A. Gatti, J. Mod. Opt. 55, 2025 (July 2008).

[23] N. Treps and C. Fabre, Phys. Rev. A 62, 033816 (2000).

[24] A. Ourjoumtsev, R. Tualle-Brouri, J. Laurat, and P. Grangier, Science 312, 83 (2006). 